\title{
Desenvolvimento e Caracterização de Sensor de Acúmulo de Dose de Radiação Azul Polimérico
}

\author{
Cláudia K. B. de Vasconcelos, Giovana R. Ferreira, Rodrigo F. Bianchi \\ Departamento de Física do Instituto de Ciências Exatas e Biológicas, UFOP
}

\begin{abstract}
Resumo: Neste trabalho as propriedades ópticas do poli[2-metóxi,5-(2'etil-hexiloxi)-p-fenilenovinileno] - MEH-PPV foram estudadas sob efeito de luz azul proveniente de super LEDs utilizada no tratamento da hiperbilirrubinemia neonatal (ou icterícia). Os resultados mostraram que o MEH-PPV apresenta uma mudança de cor do vermelho-alaranjado para o incolor, passando por diversas tonalidades de amarelo, bem como uma diminuição da intensidade dos espectros de absorção e de fotoluminescência (PL) em função do tempo de exposição à radiação. Essas mudanças foram usadas para projetar um sensor de acúmulo de dose de radiação azul, tipo indicador de cores, para avaliar a dose de radiação recebida por recém-nascidos durante o tratamento da icterícia neonatal.
\end{abstract}

Palavras-chave: Dosimetria, fototerapia, polímeros eletrônicos, icterícia.

\section{Development and Characterization of a Polymer Sensor Based on the Accumulation of Blue Light Dose}

\begin{abstract}
In this work we investigated the optical behavior of poly[2-methoxy-5(2'-ethylhexyloxy)-p-phenylenevinylene] (MEH-PPV) under the effect of blue-light LED source applied in neonatal hyperbilirubinemia treatment. There is a visible response that covers the electronic absorption of bilirubin $(350-500 \mathrm{~nm})$, and hence this material is applicable for controlling the radiation doses in the treatment of jaundice of neonates, which is one of the most common reasons for hospital readmission of newborn infants. The results show that the material presents a gradation of color from orange-red to yellow and quenching on the absorption and photoluminescent (PL) spectra with the radiation exposure time. The rate of these changes can be altered by manipulations of organic solution concentration, suggesting these color and emission/ absorption changes can be used to design an indicator-dosimeter that is readily associated with the radiation exposure time used in treating neonatal jaundice.
\end{abstract}

Keywords: Dosimetry, phototherapy, electronic polymers, jaundice.

\section{Introdução}

Nos últimos anos os polímeros conjugados passaram a ser amplamente utilizados como elementos ativos de dispositivos emissores de luz (polymer light-emitting diodes - PLEDs) ${ }^{[1]}$, não somente pela facilidade de processamento, mas também pelo baixo custo de produção, pela flexibilidade, pela possibilidade de construção de painéis luminosos com grande área, pelo custo relativamente baixo quando comparado ao custo de preparação dos tradicionais dispositivos inorgânicos e finalmente, pelo fato de que muitas aplicações destes materiais ainda estarem em fase de protótipos ou em estágio inicial de pesquisa científica. Entretanto, devido a sua natureza conjugada, estes polímeros são susceptíveis a processos de degradação que alteram drasticamente sua emissão e reduzem assim a eficiência e a durabilidade dos seus dispositivos ${ }^{[1,2]}$. Neste âmbito, fenômenos responsáveis pela durabilidade e pela eficiência desses sistemas ainda são pouco compreendidos ou controlados ${ }^{[3]}$ e, por esse motivo, muitas são as limitações com que os PLEDs se defrontam para adentrarem num mercado cada vez mais competitivo. Esse é o caso, por exemplo, dos efeitos da fotoxidação, uma das principais causas dessa restrição $0^{[4]}$. Todavia, se do ponto de vista científico e de tecnologia de dispositivos emissores de luz a fotoxidação dos polímeros luminescentes é um fenômeno que deve ser investigado, compreendido e minimizado, do ponto de vista tecnológico este fenômeno se destaca como uma importante característica para o desenvolvimento de novos dispositivos eletrônicos, tais como sensores para radiação ionizante ou não, onde a variação das propriedades ópticas dos materiais poliméricos com diferentes fontes e doses de radiação é mais importante do que a otimização da eficiência luminosa e do tempo de vida dos seus dispositivos eletroluminescentes.

Em paralelo a preocupação dos cientistas das áreas de polímeros e de engenharia de dispositivos com a necessidade de controle e minimização dos efeitos de fotoxidação em PLEDs, na área médica há atualmente um grande apelo para o desenvolvimento de sensores para monitoramento de doses de radiação aplicadas em tratamentos terapêuticos

Autor para correspondência: Cláudia K. B. de Vasconcelos, Departamento de Física, UFOP, CEP: 35400-000, Ouro Preto, MG, Brasil. 
e não evasivos, como por exemplo, na fototerapia neonatal empregada na redução da concentração sérica de bilirrubina no sangue de pacientes com hiperbilirrubinemia (ou icterícia neonatal $)^{[5]}$. Tal doença é uma das patologias neonatais que têm recebido grande atenção nos últimos anos, não somente porque cerca de $80 \%$ dos recém-nascidos prematuros apresentam essa doença ${ }^{[6]}$, mas, sobretudo, porque é uma das causas mais comuns de alta tardia e também porque pode levar os neonatos a danos neurológicos definitivos ou até mesmo a morte ${ }^{[7]}$. Embora seu tratamento seja extremamente simples, por meio da exposição dos doentes à luz visível na faixa de comprimento de onda de 425 a $475 \mathrm{~nm}$, estudos clínicos mostram a existência de dois fatores majoritários que influenciam diretamente na eficácia do tratamento, a saber $^{[6,8,9]}$ : i) o espectro da luz emitida; e ii) a dose total de luz recebida pelo neonato, uma vez que a eficiência deste tipo de tratamento tem relação direta com a superfície corporal do recém-nascido exposta à radiação luminosa e também com a distância da fonte de luz utilizada pelo equipamento de fototerapia. Como consequiência desses fatores, o tratamento se faz ineficaz, o que prejudica o controle e o diagnóstico das verdadeiras causas da doença ${ }^{[9]}$. Infelizmente, segundo relatado por Vieira et al. ${ }^{[10]}$, este fatores de controle hospitalar de responsabilidade dos profissionais da área de saúde é uma das principais causas da ineficácia do tratamento. Como resultado faz-se então necessário o monitoramento dos espectros de radiação dos equipamentos de fototerapia e das doses de radiação absorvidas pelos neonatos no intuito de aumentar a eficiência deste tipo de tratamento ${ }^{[9]}$. Logo, associar as mudanças de propriedades ópticas de polímeros luminescentes, devido aos efeitos da radiação, com aplicações em áreas médicas e/ou hospitalares é um tema interessante, atual e bastante oportuno. Em suma, estudar o comportamento das propriedades ópticas de polímeros luminescentes quando expostos a diferentes fontes e doses de radiação não é somente uma questão em aberto na literatura e que merece especial destaque no âmbito científico, mas uma oportunidade para o desenvolvimento de novos dispositivos onde a degradação da camada polimérica torna-se, nesse caso, um das suas principais vantagen ${ }^{[11,12]}$ Neste trabalho é apresentado o desenvolvimento e a caracterização de um sensor de acúmulo de dose de radiação azul polimérico para o controle da fototerapia neonatal. Inédito e de baixo custo, esse sensor ainda apresenta facilidade de fabricação e leitura de dose individual para o monitoramento e controle da fototerapia neonatal.

\section{Experimental}

Neste trabalho foram utilizadas soluções de MEH-PPV (Sigma Aldrich) em clorofórmio com concentrações entre 10 e $1000 \mu \mathrm{g} \cdot \mathrm{mL}^{-1}$. Para analisar o papel da luz e do oxigênio no processo de degradação da cadeia polimérica, que age preferencialmente no sentido de substituir ligações vinílicas $(\mathrm{C}=\mathrm{C})$ por carbonilas $(\mathrm{C}=\mathrm{O})$ na cadeia polimérica principal $^{[1,2]}$, algumas soluções foram enriquecidas, por 20 minutos com gás oxigênio $\left(\mathrm{O}_{2}\right)(99 \%)$, enquanto outras foram enriquecidas com gás nitrogênio $\left(\mathrm{N}_{2}\right)(99,99 \%)$, essa última para eliminar traços de $\mathrm{O}_{2}$ presentes na solução. Os sistemas, uma vez preparados, foram expostos a diferentes doses $\left(0\right.$ a $\left.1100 \mathrm{~J} / \mathrm{cm}^{2}\right)$ de radiação azul (foco em $460 \mathrm{~nm}$ ) proveniente de um sistema comercial de fototerapia neonatal composto por 5 super LEDs azuis, mantendo-se, para tanto, as mesmas condições usadas em tratamentos hospitalares ${ }^{[9,13]}$. Durante o procedimento de irradiação, soluções com $2 \mathrm{~mL}$ foram mantidas em ampolas de vidro, cujas pontas capilares foram lacradas por um maçarico para evitar a evaporação do solvente. As ampolas contendo as soluções de MEH-PPV, por sua vez, foram caracterizadas por meio de espectroscopia de absorção do ultravioleta visível (UV-VIS) e de fotoluminescência com o auxílio, respectivamente, de um espectrofotômetro UV-VIS SHIMADZU-1650 e de um espectrofotômetro OCEAN OPTICS USB2000 acoplado a um LED azul ( $\lambda_{\text {máx }}=460 \mathrm{~nm}, 5 \mathrm{~mm}, 2000 \mathrm{mcd}$ ), que foi usado como fonte de excitação das amostras. Para a identificação das cores foi utilizado o colorímetro portátil Pantone ${ }^{\circledR}$ Color $\mathrm{Cue}^{\circledR} 2$ e a escala Pantone de cores e a partir das coordenadas cromáticas obtidas foram então confeccionados Diagramas de Cromaticidade CIE (1931).

\section{Resultados e Discussões}

A Figura 1a apresenta os espectros de absorção para soluções de $50 \mu \mathrm{g} \cdot \mathrm{mL}^{-1}$ expostas a diferentes doses de radiação azul e enriquecidas com $\mathrm{N}_{2}$, enquanto a Figura $1 \mathrm{~b}$ apresenta os espectros de absorção para soluções de mesma concentração agora enriquecidas com $\mathrm{O}_{2}$.

Com base nos resultados mostrados na Figura 1, observa-se que os espectros das soluções diminuem a intensidade de seus picos (ou máximos) de absorção e se deslocam para menores comprimentos de onda a medida que são irradiadas. Para as amostras enriquecidas com $\mathrm{O}_{2}$, Figura $1 \mathrm{~b}$, observase que essa variação é mais rápida do que a observada para as amostras enriquecidas com $\mathrm{N}_{2}$, Figura 1a, demonstrando a influência do oxigênio e da dose de radiação na resposta óptica da solução polimérica. Nesse sentido, e a título de ilustração, a Figura 2 mostra o sistema constituído pelas ampolas de vidro preenchidas com a solução do polímero luminescente e submetidas a diferentes tempos de exposição à radiação. Nesse caso, a coloração do material muda do vermelho para o incolor, passando pelo laranja e depois pelo amarelo, à medida que a amostra é exposta à radiação.

A Figura 3a apresenta os espectros de fotoluminescência para as mesmas soluções apresentadas na Figura 2 (50 $\mu$ g. $\mathrm{mL}^{-1}$ ) enriquecidas com $\mathrm{N}_{2}$, enquanto a Figura $3 \mathrm{~b}$ apresenta os espectros de fotoluminescência para soluções de mesma concentração, mas enriquecidas com $\mathrm{O}_{2}$.

Dos resultados apresentados na Figura 3, observa-se que, assim como para os gráficos de absorção (Figura 1), existe um deslocamento do pico de máxima emissão para menores 


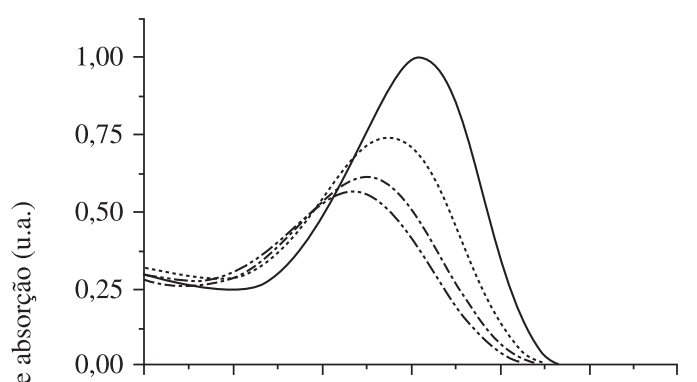

(a)

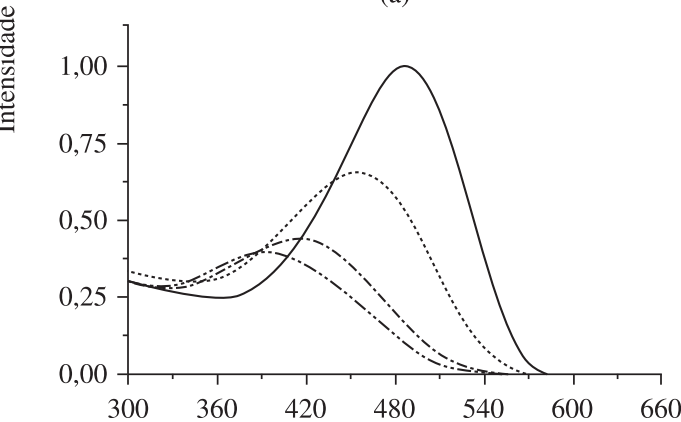

(b)

Comprimento de onda (nm)

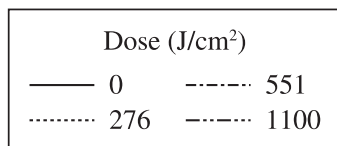

Figura 1. Espectros de absorção para soluções de $\mathrm{MEH}-\mathrm{PPV}$ em $\mathrm{CHCl}_{3}$ $\left(50 \mu \mathrm{g} \cdot \mathrm{mL}^{-1}\right)$ enriquecidas com a) $\mathrm{N}_{2}$; e b) $\mathrm{O}_{2}$.

comprimentos de onda com a dose de radiação. Entretanto, durante os primeiros minutos de exposição à radiação, há um aumento significativo na intensidade de fotoluminescência para as duas soluções. Para a solução enriquecida com $\mathrm{N}_{2}$, Figura 3a, um máximo de fotoluminescência é atingido após a dose de $276 \mathrm{~J} / \mathrm{cm}^{2}$, enquanto que para a solução enriquecida com $\mathrm{O}_{2}$, Figura $3 \mathrm{~b}$, este máximo é atingido após a dose de $92 \mathrm{~J} / \mathrm{cm}^{2}$. Este aumento pode estar relacionado possivelmente a: i) um processo de "fotopolimerização", ou seja, uma polimerização induzida por luz em virtude da etapa final do processo de polimerização (eliminação) em algumas porções da cadeia polimérica não ter sido realizada por completo; e/ou ii) uma eficiente difusão espectral de cargas excitadas dentro da cadeia polimérica ainda não degradada por um processo de transferência de energia Förster como já relatado na literatura ${ }^{[14]}$.

Vale ressaltar que o deslocamento observado dos máximos de absorção e fotoluminescência para menores comprimentos de onda observados nas Figuras 1 e 3, bem como da diminuição da intensidade desses espectros, são característicos dos processos de fotoxidação da cadeia polimérica principal do MEH-PPV que levam à substituição das ligações vínilicas $(\mathrm{C}=\mathrm{C})$ por carbonilas $(\mathrm{C}=\mathrm{O})^{[1,2]}$. Ainda nesse sentido, observa-se claramente que, além das mudanças nos espectros de absorção e de fotoluminescência, a cor da solução é alterada de acordo com a dose de radiação azul e com a concentração polimérica utilizada. Para se correlacionar mudanças de cor

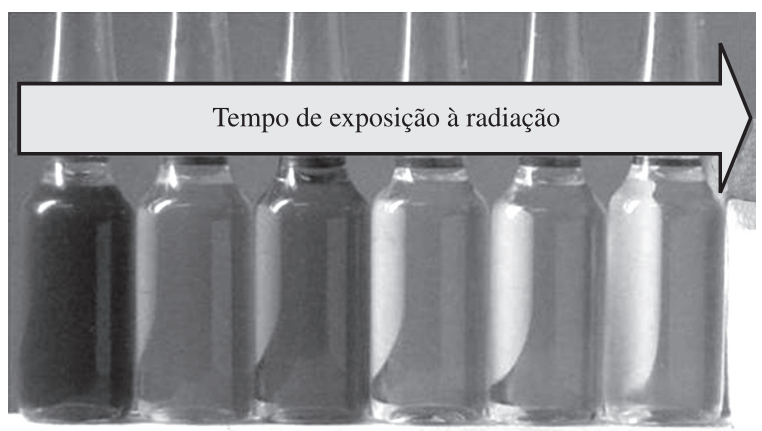

Figura 2. Sistema constituído de ampola e soluções de $\mathrm{MEH}-\mathrm{PPV}$ em $\mathrm{CHCl}_{3}$ utilizada como sensor de radiação azul. A coloração da solução polimérica muda do vermelho para o incolor, passando pelo laranja e pelo amarelo, à medida que a solução vai sendo exposta à radiação azul.

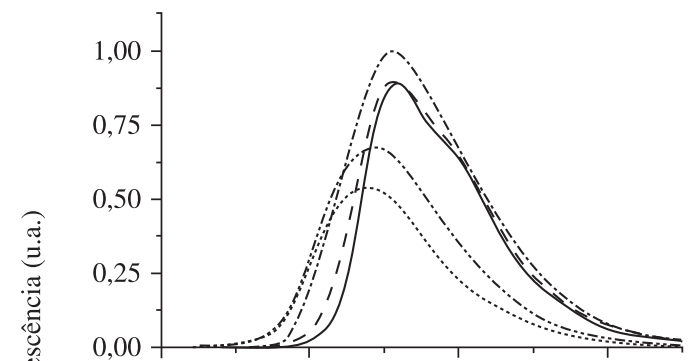

(a)

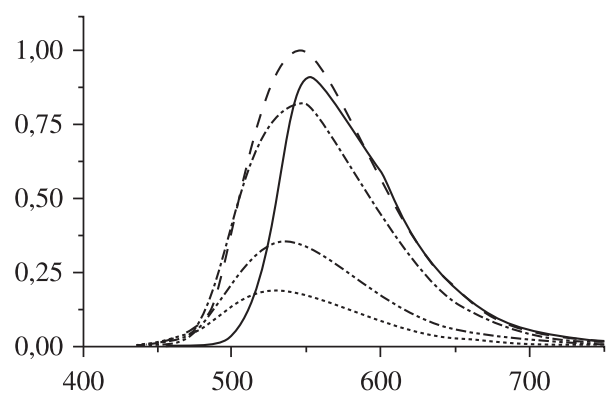

(b)

Comprimento de onda (nm)

\begin{tabular}{|c|c|}
\hline \multicolumn{2}{|c|}{ Dose $\left(\mathrm{J} / \mathrm{cm}^{2}\right)$} \\
\hline-0 & $-\cdots-551$ \\
\hline---92 & -........ 1100 \\
\hline$-\cdot-\cdot \cdot 276$ & \\
\hline
\end{tabular}

Figura 3. Espectros de fotoluminescência para soluções de MEH-PPV em $\mathrm{CHCl}_{3} 50 \mu \mathrm{g} \cdot \mathrm{mL}^{-1}$ enriquecidas com a) $\mathrm{N}_{2}$; e b) $\mathrm{O}_{2}$.

com a dose de radiação absorvida e com a concentração em massa do polímero, o padrão "X Y" de cores foi obtido com um espectro-colorímetro portátil pré-programado (Pantone ${ }^{\circledR}$ Color $\mathrm{Cue}^{\circledR}$ 2) para as cores das soluções com diferentes concentrações. Os resultados foram descritos por meio do diagrama CIE (1931) de coordenadas cromáticas apresentado na Figura 4.

Com base nos resultados apresentados na Figura 4 observa-se que a taxa de variação dos pontos de coordenadas cromáticas no diagrama CIE (1931) depende tanto da concentração polimérica como da dose de radiação incidente. Nesse caso, as amostras mais diluídas tornam-se incolores mais rapidamente do que as soluções mais concentradas e 


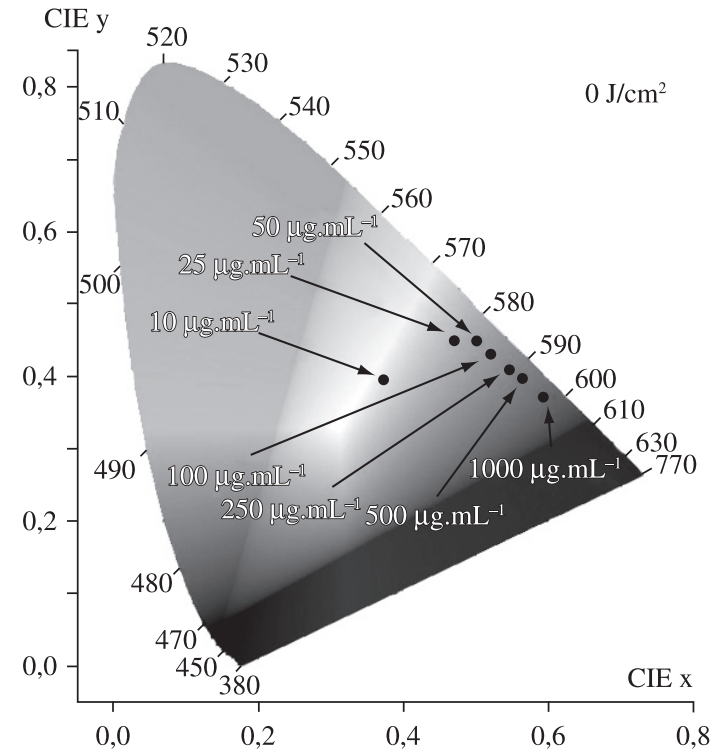

(a)

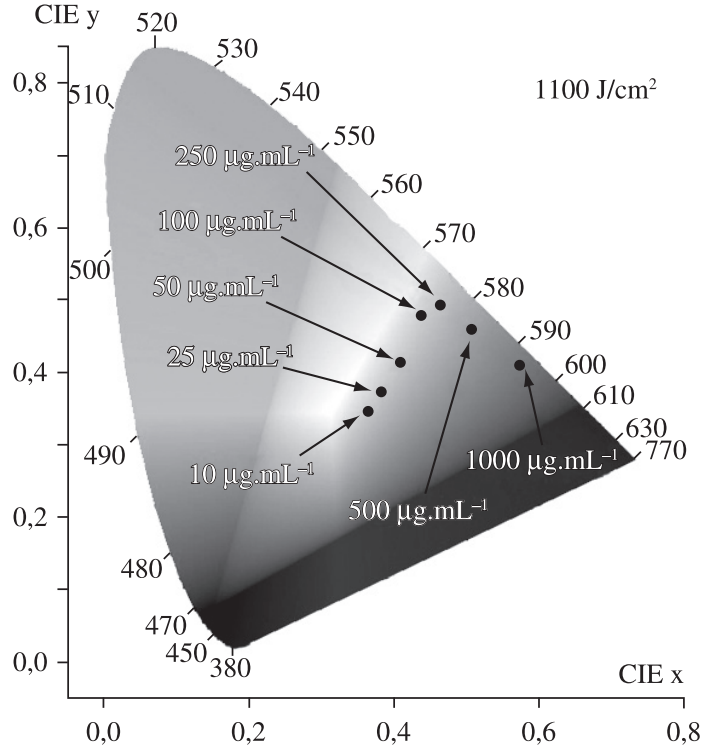

(b)

Figura 4. Diagramas CIE (1931) de cromaticidade para soluções de MEH-PPV com diferentes concentrações expostas a diferentes doses de radiação azul a) $0 \mathrm{~J} / \mathrm{cm}^{2} ;$ e b) $1100 \mathrm{~J} / \mathrm{cm}^{2}$.

todas caminham no sentido vermelho - amarelo - incolor com a radiação. Além disso, amostras mais diluídas, de acordo com os efeitos de solvatocromismo ou de concentração, apresentam-se mais amareladas do que as amostras mais concentradas. Outro ponto que pode ser levantado a partir dos diagramas CIE (1931) de coordenadas cromáticas é a diagramação destes resultados por meio de uma tabela de cores. A Figura 5 apresenta essa tabela juntamente com a referência na escala Pantone, um padrão internacional de cores para artes gráficas. Esse padrão permite um controle

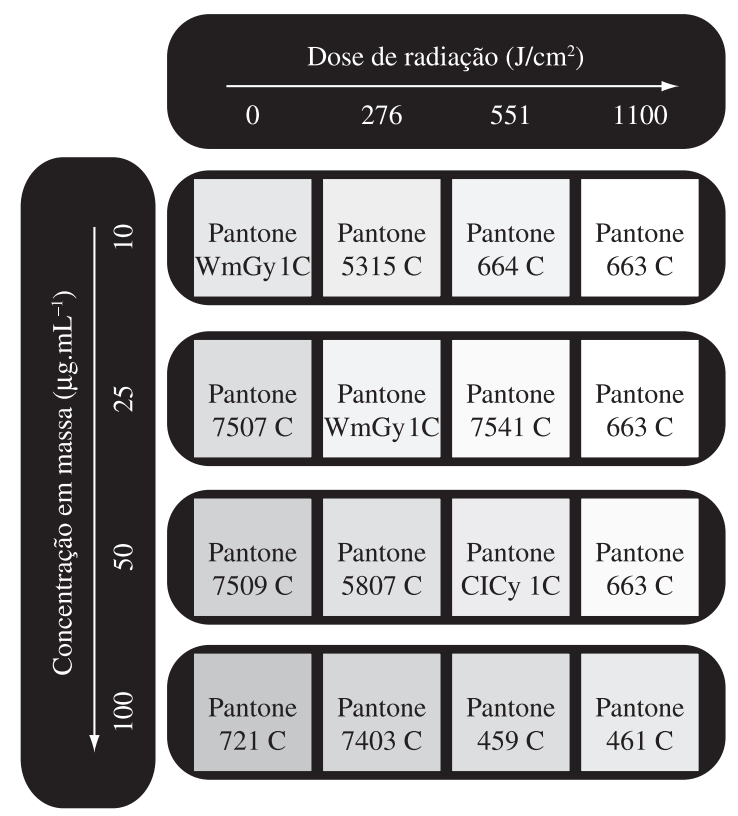

Figura 5. Tabela de cores padrão identificada com a referência correspondente à escala Pantone de Cores. A tabela também apresenta os respectivos valores de dose de exposição do polímero à radiação azul e as concentrações em massa utilizadas. mais preciso da cor e, portanto, que as impressões gráficas das tabelas sejam fieis às cores das soluções poliméricas irradiadas ou não. Nessa tabela fica clara a correlação entre cor e dose de exposição à radiação do sistema polimérico. A tabela também mostra a correlação de cor e dose com a concentração em massa da solução polimérica. Esses resultados, atrelado aos já apresentados por Vasconcelos \& Bianchi ${ }^{[15]}$, mostram que a manipulação tanto da concentração das soluções poliméricas como da presença de $\mathrm{O}_{2}$ e $\mathrm{N}_{2}$ é de grande importância para o desenvolvimento de sensores de acúmulo de dose de radiação azul com resposta em um amplo intervalo de tempo e/ou dose de radiação.

\section{Conclusões}

Os resultados obtidos nesse trabalho mostram a evolução das cores e dos espectros de absorção e de fotoluminescência de soluções à base de MEH-PPV como função da dose de exposição à radiação azul. Por meio da manipulação da concentração das soluções poliméricas observou-se que o processo de degradação do polímero está intimamente relacionado à concentração em massa utilizada, ou seja, maiores concentrações de MEH-PPV levam a taxas de degradação mais lentas. Outro fator de fundamental importância está relacionado à presença de $\mathrm{O}_{2}$ ou $\mathrm{N}_{2}$ na solução polimérica preparada. A presença de oxigênio no sistema polimérico leva a uma aceleração do mecanismo de degradação do polímero, bem como a diminuição do tempo necessário para se atingir um máximo de fotoluminescência para concentrações superiores a $50 \mu \mathrm{g} \cdot \mathrm{mL}^{-1}$. Vale ainda salientar que o sensor proposto para o monitoramento da radiação empregada na terapêutica da hiperbilirrubinemia é um sistema inovador de controle de qualidade dos 
equipamentos de fototerapia neonatal. Outro aspecto que merece destaque é o fato de que este sensor apresenta leitura de dose rápida (por meio da comparação com uma tabela de cores pré-existente), facilidade de processamento e manufatura, possibilidade de criação de um banco de dados, confiabilidade, estabilidade, facilidade de manuseio e leitura, segurança e baixo custo, ou seja, requisitos essenciais para o seu uso por profissionais de saúde com as mais diversas formações, como enfermeiras, médicos etc.

\section{Agradecimentos}

Os autores agradecem o CNPq, INEO/CNPq, a FAPEMIG e a CAPES pelo suporte financeiro.

\section{Referências Bibliográficas}

1. Burroughes, J. H.; Bradley, D. D. C.; Brown, A. R.; Marks, R. N.; Mackay, K.; Friend, R. H.; Burns, P. L. \& Holmes, A. B. - Nature, 347, p.539 (1990).

2. Argyrakis, P.; Kobryanskii, M. V.; Sluch, M. I. \& Vitukhnovsky, A. G. - Synth. Met., 91, p.159-160 (1997)

3. Cao, Y.; Parker, I. D.; Yu, G.; Zhang, C. \& Heeger, A. J. - Nature, 397, p.414-417 (1999).

4. Bianchi, R. F.; Balogh, D. T.; Tinani, M.; Faria, R. M. \& Irene, E. A. - J. Polymer Sci. Polymer Phys., 42, p.1033-1041 (2004).

5. Phyllis, A.; Dennery, M. D.; Daniel, S.; Seidman, M. D.; David, K. \& Stevenson, M. D. - N. Engl. J. Med., 344, p.581-590 (2001).
6. Grossweiner, L. I.; Grossweiner, J. B. \& Rogers, BHG. "The science of phototherapy: an introduction", Springer, New York, 374 p. (2005).

7. Ramos, J. L. A. - Rev. Fac. Cienc. Med. Sorocaba, 4, p.17-30 (2002).

8. Silva, F. M. D.; Ideriha, E. T. E. \& Netto, E. J. "Desenvolvimento de sistema óptico para unidade de fototerapia neonatal", in: Anais Memorias del II Congreso Latinoamericano de Ingeniería Biomédica, Cuba, may (2001).

9. Maisels, M. J. \& McDonagh, A. F. - N. Engl. J. Med., 358, p.920-928 (2008).

10. Vieira, A. A.; Lima, C. L. M. A.; Carvalho, M. \& Moreira, MEL. - Rev. Bras. Saúde Mater. Infant., 4, p.359-366 (2004).

11. Vasconcelos, C. K. B. \& Bianchi, R. F. - Polímeros Cienc Tecnol., 17, p.325-328 (2007).

12. Ferreira, G. R.; Vasconcelos, C. K. B. \& Bianchi, R. F. Med. Phys., 36, p.642 (2009).

13. Tan, K. L. - J. Pediatr., 90, p.448-452 (1977).

14. Anni, M.; Gigli, G.; Cingolani, R.; Gobato, Y. G.; Vercik, A.; Marletta, A.; Guimarães, F. G. E. \& Faria, R. M. - Phys. Rev. Condens. Matter Mater. Phys., 68, 035215.1-035215.6 (2003).

15. Vasconcelos, C. K. B. \& Bianchi, R. F. - Sensor. Actuator B Chem., 143, p.30-34, (2009).

Enviado: 15/08/09

Reenviado: 27/09/09

Aceito:22/10/09

DOI: $10.1590 / \mathrm{S} 0104-14282010005000010$ 